\title{
La evolución educativa universitaria y el proceso de innovación en la enseñanza pública ecuatoriana. La innovación es desarrollo social
}

\section{The educational evolution of the university and the process of innovation in Ecuadorian public education. Innovation is social development}

\author{
MSc, Norma Narcisa Garcés Garcés ${ }^{1}$ \\ norma.garcesg@ug.edu.ec \\ Dr, Roosevelt Barros Morales ${ }^{2}$ \\ roosevelt.barrosm@ug.edu.ec
}

Recibido: 1/04/2018, Aceptado: 1/06/2018

\begin{abstract}
RESUMEN
El objetivo de la investigación es adentrarse en la evolución de la educación superior ecuatoriana desde 1830 hasta la actualidad, en que desde el 2007 se instituyó una revolución educativa, permitiendo que los docentes alcancen capacitación permanente, la remuneración de los docentes sea revisada y mejore las condiciones laborales y profesionales. Se estudió la calidad de la educación superior en la educación, la innovación de la calidad educativa como vía para implementar nuevas formas de evaluación educativa, los procesos transformadores de la administración educativa a nivel superior y la propuesta para la universidad del futuro como la formación pedagógica de los docentes, reconocimiento de la tarea del docente, superar la burocratización y complejidad administrativa, se propone un cambio del sistema a través de la reducción burocrática, trabajar en un nuevo modelo pedagógico, cambiar el sistema de transferencia de conocimientos, basado en la clase magistral, para plantear otras opciones educativas basada en el dialogo, intercambios y las actividades prácticas, las clases virtuales con la forma de clase invertida, mejorar la actitud e incrementar la colaboración, se apuesta por un incremento en la motivación del profesorado y la valoración de los individuos en relación a la innovación y el cambio, transversalidad, actualización y flexibilización de las titulaciones, es necesario generar nuevos modelos pedagógicos y formatos educativos que tengan en cuenta las más recientes investigaciones que ofrecen puntos de reflexión sobre el proceso de enseñanza aprendizaje, factor emocional, simplificación de los procesos internos a la institución y mayor autonomía para favorecer la diversificación.
\end{abstract}

\footnotetext{
1 Docente - Investigadora -Consultora académica Facultad de Filosofía, Letras y Ciencias de la Educación Universidad de Guayaquil, Ecuador

2 Director del Dpto. Investigación Universidad de Guayaquil Facultad de Filosofía, Letras y Ciencias de la Educación, Ecuador
} 
Palabras clave: Evolución educativa, innovación, enseñanza pública

\begin{abstract}
The objective of the research is to delve into the evolution of higher education in Ecuador from the time of 1830 to the present, in which since 2007 an educational revolution was instituted, allowing teachers to achieve permanent training, remuneration of and improve working and professional conditions. The quality of higher education in education, the innovation of educational quality as a way to implement new forms of educational evaluation, the transformative processes of educational administration at the higher level and the proposal for the university of the future as pedagogical training of teachers, recognition of the teacher's task, overcoming bureaucratization and administrative complexity, proposes a change of the system through bureaucratic reduction, work on a new pedagogical model, change the system of transfer of knowledge, based on class magistral, to raise other educational options based on dialogue, exchanges and practical activities, virtual classes with the form of inverted class, improve attitude and increase collaboration, is committed to an increase in teacher motivation and assessment of Individuals who already have a positive attitude regarding innovation and change, transversality, updating and flexibility of qualifications, it is necessary to generate new pedagogical models and educational formats that take into account the most recent research that offer points of reflection on the Process of teaching learning, emotional factor, simplification of the internal processes to the institution and greater autonomy to favor the diversification.
\end{abstract}

Keywords: Educational evolution, innovation, public education

\title{
Introducción
}

La historia del Ecuador, en el año 1830, cuando se organizó la república soberana e independiente se consagró promover a la educación pública como un derecho inalienable de las personas para el desarrollo humano y proyecto de vida, en las prescripciones constitucionales, se promovió fundamentar la instrucción pública, plantear programas generales y dictar leyes de enseñanzas para todas las instituciones públicas y privadas, además se estableció la primara de carácter oficial que sea la enseñanza gratuita y obligatoria.

Este documento que se puso en marcha para el beneficio de los ciudadanos y el motivo de mejorar la educación del país y dar apertura a otro tipo de enseñanza impartida por las religiosas, que primera instancia fue beneficioso para el pueblo ecuatoriano porque era una educación tradicionalista, estricta, religiosa, moralista y sistemática; en cambio la educación actual es flexible, convergente abierta, conocedora de la problemática actual para desarrollar competencias apropiadas que realmente sean transformadoras y emprendedoras.

\section{Desarrollo}

La innovación educativa dentro del país tuvo un cambio radical a partir del 2007 hasta nuestra actualidad, las manifestaciones dentro de los grupos sociales fueron caracterizadas por la mayoría de los ciudadanos y tomadas en cuenta del actual gobierno, incentivando a la preparación intelectual y a la facilidad de su libre ingreso para la mejora dentro de la sociedad ecuatoriana. Los gobiernos anteriores tanto Bucaram, Mahuad, Gutiérrez y Noboa, generaron gran insatisfacción y descontentos dentro de la sociedad ecuatoriana, por cuanto el manejo de la economía no era coherente con las demandas del pueblo. La educación está en un desequilibrio y en caos porque era manejado y manipulado por estos gobernantes que solo los usaban 


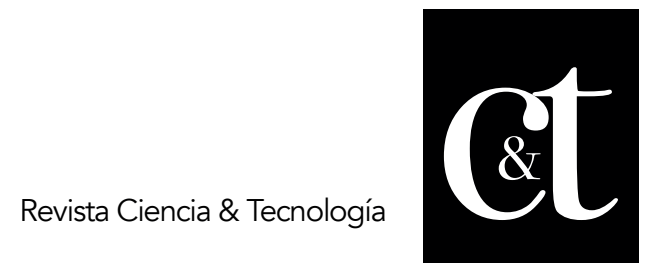

No. 19, 31 de julio de 2018

ISSN impreso: 1390 - 6321

como mitin político para generar votos a favor o para desestabilizar los gobiernos de turnos en muchos casos, creando resistencia en los docentes

La reacción de aquellos gobiernos se vio reflejada en la persecución, prisión y la escasa nula apertura a las propuestas de los maestros y entra en el juego el magisterio que viene a ser la fuerza de resistencia a los gobiernos y como menciona Rene Ramírez en su artículo Transformaciones de la Educación Superior comenzó a correr la legitimidad de los gobiernos...el cambio inminente exigido por la persistente protesta popular...,las alianzas entre el frente indígena y los sectores de centroizquierda... exigía cambios visibles que solo eran posibles en la democracia (Ramírez, 2013).

Al consolidarse la democracia se elige presidente de la República del Ecuador al Econ. Rafael Correa Delgado, periodo de 10 años que reflejan una década de cambios profundos y estructurales en el sistema político, económico, social y educativo ecuatoriano, la gratuidad de la educación en el ecuador desde la inicial hasta la universitaria es uno de los logros de mayor reconocimiento a su gestión, al igual que la auditoria universitaria llevada a cabo tanto en universidades públicas y privadas para ubicarlas de acuerdo a la infraestructura, calidad de docentes, producción científica y los diversos sistema de evaluación que se implementaban para categorizarlas o intervenirlas para su mejoramiento continuo o re categorizarlas de acuerdo a las observaciones cumplidas y ser reconocidas por su visión y acción nacional e internacional integradora de saberes.

Los cambios favorables en la educación dentro del Ecuador Podemos definir que dentro de la educación ecuatoriana se establecieron algunas metas que por diferentes índoles algunas se pudieron cumplir y otras quedaron inconclusas en su debido proceso, las metas se caracterizaron por metas llamadas Educación para todos y sus faces comprendían de la siguiente manera como nos indica Bramwell (2015):

En el año 2000, en el Foro Mundial de Educación realizado en Dakar, el Ministerio de Educación del Ecuador ratificó el compromiso con las seis metas de Educación para Todos (EPT) a cumplirse hasta el año 2015, al igual que lo hicieron la mayoría de país de región y el mundo.

Estas metas buscaban incrementar (1) la atención a la primera infancia, (2) el acceso a la educación primaria de calidad, (3) el acceso equitativo de jóvenes a programas apropiados de aprendizaje, (4) los niveles de alfabetismo y el acceso a educación para adultos, (5) la equidad de género y (6) la calidad de la educación (UNESCO, 2003). Al acercarnos a 2015, la UNESCO evaluará el nivel de cumplimiento de las metas de EPT en todo el mundo, y también publicará casos de países que han tenidos logros considerables en una o más de las metas de EPT, como es el caso de Ecuador.

El sistema Educativo universitario desde el 2008 con la aprobación de la nueva Constitución de la República del Ecuador en la que se puntualizaba que la educación superior es considerada de interés público sin fines de lucro y determinada como un derecho inalienable e inexcusable de todos los seres humanos y que el estado asumía el control, supervisión, acción y regulación de la educación en todos los niveles. 


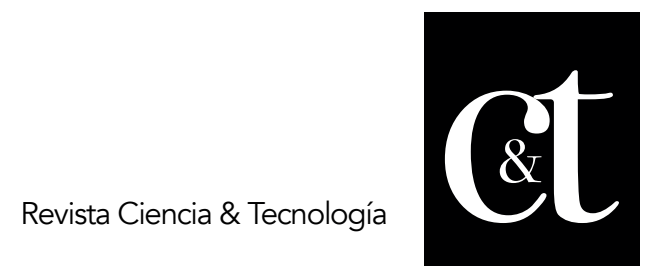

No. 19, 31 de julio de 2018

ISSN impreso: 1390 - 6321

\section{Niveles}

La propuesta que fortaleció con la aprobación de la nueva constitución del Ecuador del 2008 en el que garantizaba como un derecho de las personas a lo largo de toda su vida y un deber inalienable e inexcusable del Estado) articulo 26. Se estableció mecanismos legales como la ley de Educación Superior del Ecuador, reglamento de la educación superior para garantiza la calidad de la educación y además que el estado garantizara una buena remuneración para el magisterio, capacitación permanente, establecimiento de un sistema de evaluación del docente, directivo, infraestructura y la Recategorización del docente.

En la nueva ley Orgánica de Educación superior se dio apertura para la creación de nuevas instituciones de educación superior como el YACHAY para suplir la formación técnico superior de los docentes que estaban a cargo de los institutos superiores pedagógicos de educación, los mismos que a partir de su evaluación dejaron de funcionar al igual que la formación en educación en las universidades públicas.

A la par continúa las facultades de filosofía con las carreras tradicionales en pedagogía hasta cumplir con los estudiantes matriculados para aquellas.

En el año 2013 se evaluó a los 15 institutos superiores de artes y conservatorios superiores - ISACS. Con la elaboración del modelo de evaluación en febrero del 2013, se emitieron informes sobre las universidades que aparecieron con sedes y extensiones localizadas a nivel nacional y que no llenaba las expectativas de una educación de calidad. Estas universidades fueron calificadas y ubicadas en la categoría E calificación más baja, en conclusión, significaba que el desempeño institucional académico de dichas universidades era deficiente y en algunos casos se había convertido en negocios familiares que ofrecían poco estudio y un alto precio por los estudios.

A partir de la evaluación de las carreras se propone el rediseño de estas el que se somete a la evaluación y monitoreo del Consejo de evaluación, acreditación y aseguramiento de la calidad de la educación superior Ceaaces y que responde a la realidad educativa del Ecuador, esta se convierte en un tema de discusión abierta en todo el país y una búsqueda de una educación superior que se alinee a las demandas y expectativas que requiere el país para su desarrollo.

El modelo educativo planteado por el Ceaaces puntualiza dos componentes macro, primero el entorno de aprendizaje, y que prioriza la evaluación institucional de la universidad y que refleja la calidad de la educación en lo referente a infraestructura, planta de docentes, gestión del patrimonio. El segundo componente son los resultados de aprendizaje y se refiere a la formación del estudiante Para determinar la calidad de la educación se trazaron más de 100 indicadores que al final del proceso se redujeron a 47 . Se priorizaron dos grandes componentes el de la academia y el de la calidad del docente.

La academia que enfatiza el quehacer académico, la carrera universitaria, contrataciones, remuneraciones, escalafones y derechos. En cambio, la calidad de docente instruye la formación de los educadores con formación de cuarto nivel como maestrías y doctorados, el tipo de carreras ofertadas, y su nivel de especialización, además se consideró entre indicadores el número y la cantidad de bibliotecas que tienen una institución, estructuras y campo, la gestión o de gerencia educativa 
El Reglamento del Consejo de Educación Superior nos indica: Que, el artículo 166 de la Ley ibídem, establece: "El Consejo de Educación Superior es el organismo de derecho público, con personería jurídica, con patrimonio propio, independencia administrativa, financiera y operativa, que tiene por objetivo la planificación, regulación y coordinación interna del Sistema de Educación Superior, y la relación entre sus distintos actores con la Función Ejecutiva y la sociedad ecuatoriana (...)"

En cuanto a la educación, el texto constitucional planteado en este artículo por López (2011, pág. 380), entre otros aspectos:

- La educación es un derecho de las personas a lo largo de su vida y un deber ineludible e inexcusable del Estado. Constituye un área prioritaria de la política pública y de la inversión estatal, garantía de la igualdad e inclusión social y condición indispensable para el buen vivir. Las personas, las familias y la sociedad tienen el derecho y la responsabilidad de participar en el proceso educativo (art. 26).

- La educación se centrará en el ser humano y deberá garantizar su desarrollo holístico, el respeto a los derechos humanos, a un medio ambiente sustentable y a la democracia; obligatoria, intercultural, incluyente y diversa; impulsará la equidad de género, la justicia, la solidaridad y la paz; (...) es indispensable para el conocimiento, el ejercicio de los derechos, la construcción de un país soberano y es un eje estratégico para el desarrollo nacional (art. 27).

- La educación responderá al interés público y no estará al servicio de intereses individuales y corporativos. Se garantizará el acceso universal, permanencia, movilidad y egreso sin discriminación alguna y la obligatoriedad en el nivel inicial, básico y bachillerato o su equivalente (art. 28).

- La educación pública será universal y laica en todos sus niveles, gratuita hasta el tercer nivel de educación superior inclusive (art. 28).

- El Estado garantizará la libertad de enseñanza y cátedra y el derecho de las personas de aprender en su propia lengua y ámbito cultural (art. 29).

- La educación como servicio público se prestará a través de instituciones públicas, fiscomisionales y particulares (art. 345).

- Existirá una institución pública, con autonomía, de evaluación integral interna y externa que promueva la calidad de la educación (art. 346).

- El Estado garantizará al personal docente en todos los niveles y modalidades, estabilidad, formación continua, mejoramiento pedagógico y académico, y actualización, una remuneración justa, de acuerdo a la profesionalización, desempeño y méritos académicos. La ley regulará la carrera docente y escalafón, establecerá un sistema nacional de evaluación de desempeño y la política salarial en todos los niveles. Se establecerán políticas de promoción, movilidad y alternancia docente (art. 349).

Estos artículos recogen de gran magnitud las demandas que ha existido en nuestra sociedad que siempre fueron planteados por grandes profesionales entendidos en el ámbito educativo en el nivel superior. Su puntualiza los alcances que debe existir en todas las universidades dentro del Ecuador para implantar la línea de formadores 
profesionales.

La universidad actual ha descuidado los verdaderos roles del docente como es ensenar, educar con valores y ética, la aplicación de una malla que llene las expectativas del Ecuador de acuerdo a la matriz productiva del país, conocer los fines de la educación para la fue creada y llegar a verdaderos consensos que ayuden a fortalecer los saberes disciplinares de acuerdo a los requerimientos actuales como desarrollo tecnológico, desarrollo social, aspectos organizacionales de la convivencia social, en una realidad en que las nuevas ideas y modelos de sociedad necesitan expandirse.

Se necesita otra visión de la educación y ser muy crítico y constructivo sobre las necesidades de la escuela o la universidad. Es importante conocer las falencias, criticar y proponer para que las nuevas propuestas alcancen lo deseado.

La calidad de la enseñanza en el Ecuador Una de las propuestas de la organización iberoamericana para la educación y de la cultura es de establecer metas que deben de cumplirse en los años 2017 -2021, estos cambios sustanciales que se proponen en la educación, es buscar los objetivos claros a la inclusión de personas con capacidades especiales y asociadas como la discapacidad auditiva, visual y motora, problemas de aprendizaje. Esto se afianza a través de las generaciones de conocimientos, ya que proponen una herramienta de integralidad para lograr la superación del hombre, sin educación no hay desarrollo, por lo tanto, es necesario propiciar nuevos saberes, para dotarlo del conocimiento critico que permite discernir muchos saberes.

La calidad de la educación está influenciada en la calidad de su maestro en la preparación continua y permanente, en estructurar planes y programas nuevos en la parte educativa para llenar las expectativas de nuevas profesiones que permitan en el desarrollo profesional, la capacidad transformadora en la educación universitaria, es una deuda histórica de compromiso con millones de personas es brindar una esperanza, de responsabilidad y compromiso de educar, en libertad, igualdad y de desarrollo.

¿Cómo se determina la calidad de la educación superior en el Ecuador? Es conociendo el servicio que presta en la integración dentro de la comunidad educativa, los esfuerzos continuos del Estado por brindar infraestructura adecuada, docentes con excelencia académica, gestionar la investigación como recurso sostenible.

Según Noro (2015): Es lo que deciden ser: admite mutaciones, cambios, transformaciones. Si la escuela del pasado definía y construía a sus usuarios (alumnos, sujetos) y a los agentes (educadores) otorgándole una auténtica identidad (pág. 12).

En la Universidad de hoy, ha sufrido profundas transformaciones, en cuanto a las disciplinas que se cultivan en el modo de la identidad del desarrollo social, el ideal de cada persona que propone la ética y la moral, en el siglo XXI las formas de concebir la educación han sido diferente. Hoy en día se considera como un centro de expendedor de títulos profesionales, y da la impresión que su principal objetivo es asegurar a sus alumnos en un buen puesto de trabajo a través de la inserción del mercado laboral pero, se ha dejado de considerar los procesos educativos de 


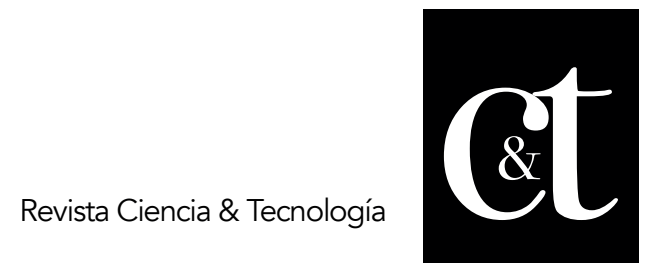

No. 19, 31 de julio de 2018

ISSN impreso: 1390 - 6321

calidad, la preparación de los profesionales del nivel superior que necesita la sociedad, médicos, doctores, maestros, ingenieros y muchas veces esta manejado por políticos que no buscan la excelencia Académica sino réditos económicos y poder y altos cargos directivos sin considerar la competitividad del mercado laboral. Y esto se presenta como un obstáculo para poder desarrollar la transparencia del conocimiento. El respeto a la identidad institucional y en definitiva la universidad es meramente administrativa.

Noro (2015) plantea: Que la escuela de hoy es una entidad que se espera en su indefinición y su definición: se construye con el aporte de los usuarios y de los autores. Es lo que deciden ser: admite mutaciones cambios y transformaciones (pág. 12).

La construcción del conocimiento se realiza a través del aporte de los estudiantes y de los docentes y de las investigaciones de expertos que ayudan a fortalecer el intelecto del hombre. Los cambios y transformaciones son preparar nuevas generaciones de líderes y aportar nuevos saberes para el progreso y desarrollo de la humanidad. La práctica de valores profundos y fuertes, capaces de despertar el respeto por el ser humano. Es ineludible pensar que ante los retos que enfrenta la universidad se resume en cinco conceptos o necesidades fundamentales para la universidad de hoy, como la relación proactiva con el medio, internacionalización, eficiencia sistemática, interdisciplinariedad y diversificación y cambio. Estos cinco términos definen, en términos generales, el nuevo modelo de la universidad que está edificando.

En cuanto del profesor Holland en la película: Adiós al profesor Holland Es la escuela tradicionalista en la que encuentra maestros con ideas obsoletas y cuando hace acto de presencia un maestro nuevo que llega a la institución son mal vistos e indispuestos, El maestro Holland incursiona en el estudiante a través de la música y ellos que no tienen el menor sentido de la música comienzan apreciar músicos trata de los sentimientos, que sale del corazón y cada día se introducen en el mundo de la música.

Los profesores tradicionalistas en vez de entusiasmar al docente nuevo tratan de minimizar y de hacerlo sentir mal y que su trabajo no sea relevante, acosadores y críticos destructivos. Tiene una maestra que todo lo ve mal, y que solo enseñe música clásica obviando el rock roll, recibe acoso laboral incluyendo otras actividades para que fracase

Los desafíos que tiene que enfrentar con un alumno que no sabe tocar ningún instrumento y que tiene que empezar de cero para integrarlo al chico en la banda, esos desafíos a los que se enfrenta el maestro, permiten dejar huellas significativas en las vidas de los discentes. Y que tiene que darle la oportunidad de destacarse para ser considerado como deportista. La gran dedicación y fe en que es un personaje con grandes dones es lo que el maestro apuesta por este alumno, utiliza cualquier método para educar el oído del estudiante.

Pasa por situaciones muy difíciles en su vida personal en la que su hijo tiene el $90 \%$ de sordera y lo que le aconsejan es que no utilicen el lenguaje de señas para que pueda encontrar su voz y comunicarse en el entorno.

Beethoven para escribir su música corta todas las partes de su vida y su oreja puso en el piano muy cerca del piano apretándola muy fuertemente aporreaba las teclas 
con las manos para poder oír su música con las vibraciones del suelo. Beethoven no era sordo de nacimiento. Estas vivencias son las que el profesor Holland las está pasando con su hijo.

La diversidad de estudiantes con mal comportamiento, cambiar las vidas de los estudiantes, el estudiante que fallece luego de haber pasado por circunstancias difíciles e incluirlo con el resto de las estudiantes. Cada estudiante es un mundo diferente que hay diferenciar las necesidades emocionales, espirituales y conocimiento científico para despertar en ellos el aprender para vivir en un estado de vida prometedor.

\section{Materiales y Métodos}

La revisión bibliográfica que se utilizó para la recopilación, procesamiento y el análisis de la información a través de bases de datos como, por ejemplo:

- La historia del Ecuador

- Los acontecimientos de la educación ecuatoriana

- Etapas de evolución de la enseñanza superior

- Los cambios favorables en la educación dentro del Ecuador

- La visión de crisis y las críticas de la educación

- Formar una propuesta para la universidad del futuro

- Los procesos transformadores de la administración educativa en el nivel superior

- La innovación en la administración educativa en el nivel superior

- La calidad de la educación superior

Los estudios exploratorios que sirven para preparar el terreno de la investigación, en el caso de la evolución educativa en el Ecuador se ha considerado documentos y evidencias de lo sucedido en el año 2007 hasta la actualidad, los pro y contra de la educación el Ecuador.

Los estudios explicativos que generan sentido de entendimiento y son estructurados en la investigación se los realiza con un estudio de campo de conocimiento específico y en el que se puede ilustrar un hecho. Los instrumentos de investigación

El estado del conocimiento en el tema de investigación que nos revele la revisión de la literatura y el enfoque que el investigador le pretenda dar a su estudio. Pero antes de ahondar en esta respuesta, es necesario hablar de cada tipo de estudio.

Los estudios exploratorios nos sirven para aumentar el grado de familiaridad con fenómenos relativamente desconocidos, obtener información sobre la posibilidad de llevar a cabo una investigación más completa sobre un contexto particular de la vida real, investigar problemas del comportamiento humano que consideren cruciales los profesionales de determinada área, identificar conceptos o variables promisorias, establecer prioridades para investigaciones posteriores o sugerir afirmaciones (postulados) verificables (Dankhe, 1986). Esta clase de estudios son comunes en la investigación del comportamiento, sobre todo en situaciones donde hay poca información.

Los estudios exploratorios en pocas ocasiones constituyen un fin en sí mismos, "por lo general determinan tendencias, identifican relaciones potenciales entre variables 
y establecen el 'tono' de investigaciones posteriores más rigurosas" (Dankhe, 1985, pág. 412). Se caracterizan por ser más flexibles en su metodología en comparación con los estudios descriptivos o explicativos, y son más amplios y dispersos que estos otros dos tipos (v.g., buscan observar tantas manifestaciones del fenómeno estudiado como sea posible). Asimismo, implican un mayor "riesgo" y requieren gran paciencia, serenidad y receptividad por parte del investigador.

Muy frecuentemente el propósito del investigador es describir situaciones y eventos. Esto es, decir cómo es y se manifiesta determinado fenómeno. Los estudios descriptivos buscan especificar las propiedades importantes de personas, grupos, comunidades o cualquier otro fenómeno que sea sometido a análisis (Dankhe, 1986). Miden o evalúan diversos aspectos, dimensiones o componentes del fenómeno o fenómenos a investigar. Desde el punto de vista científico, describir es medir. Esto es, en un estudio descriptivo se selecciona una serie de cuestiones y se mide cada una de ellas independientemente, para así -y valga la redundancia-describir lo que se investiga.

La operacionalización de las variables dependientes e independientes junto con las interrogantes de la investigación es fundamental para iniciar con la investigación y sirvió de apoyo para la investigación y que se pudo analizar la situación actual de la educación universitaria en el Ecuador y las nuevas técnicas pedagógicas innovadoras que se aplican y que se mencionan en la investigación.

Nuevas prácticas pedagógicas innovadoras: práctica flipping the classroom.

Es un modelo pedagógico que transfiere el trabajo de determinados procesos de aprendizaje que se realizan fuera del aula o mediante el sistema virtual o aula invertida y se utiliza el tiempo clase junto con la experiencia del docente estrategias nuevas para facilitar y potenciar otros procesos de adquisición y practica del conocimiento dentro del aula (The Flipped Classroom, 2015).

Esta clase es mucho más que la edición de un video o distribución de un video. Se trata de un enfoque integral que combina la instrucción que imparte el maestro de forma directa con métodos constructivistas y el compromiso y participación activa de los estudiantes con el contenido del curso y esto permite la construcción conceptual.

Este enfoque integral que cuando se aplica con éxito apoyará a todas las fases de un ciclo de aprendizaje.

Cuando los docentes diseñan y publican en línea o utilizan herramientas que compartan la información, el tiempo de clase se libera para que pueda facilitar la participación de los discentes en el aprendizaje activo a través de preguntas y discusiones y actividades aplicadas que fomentan la exploración, la articulación y aplicación de ideas.

Jonathan Bergmann y Aaron Sams, dos profesores de química en Woodland Park High School en Woodland Park Colorado, acuñaron el término "Flipped Classroom". Bergmann y Sams se dieron cuenta de que los estudiantes frecuentemente perdían algunas clases por determinadas razones (enfermedad, por ejemplo). En un esfuerzo para ayudar a estos alumnos, impulsaron la grabación y distribución de video, pero, además, se dieron cuenta que este mismo modelo permite que el profesor centre más la atención en las necesidades individuales de aprendizaje de cada estudiante. 
Instructor Peer (PI) fue desarrollado por el profesor de Harvard Eric Mazur, e incorpora una técnica denominada "enseñanza just-in-time" como un elemento complementario al modelo FC. "Enseñanza Justin-time" permite al profesor recibir retroalimentación de los estudiantes el día antes de la clase para que él puede preparar estrategias y actividades para centrarse en las deficiencias que puedan existir en los estudiantes en la comprensión del contenido.

Permite a los docentes dedicar más tiempo a la atención a la diversidad. Es una oportunidad para que los docentes compartan información y conocimiento entre sí, estudiantes, las familias y la comunidad. El ambiente de aprendizaje es colaborativo en el aula. Facilita accede a los contenidos en cualquier momento

\section{Pechakucha}

Es un formato que permite hacer una presentación a través de la elaboración de 20 diapositivas que se presentan cada una en 20 segundos. Aunque el reto no es fácil, si se trabaja con detalle la información a presentar, el PechaKucha permite presentar de manera más ágil y visual diferentes contenidos.

Se define la estructura básica, es decir se divide la materia en capítulos. Se divide 20 entre la cantidad de apartados Desarrolla los conceptos esenciales de cada apartado y a cada uno se le asigna una diapositiva., es un esquema lógico.

Probar o ensayar cada diapositiva se debe hablar o comentar en un minuto, realizar una síntesis. La crítica constructiva ayuda a pulir el trabajo.

La solución propuesta por Astrid y Mark fue tan sencilla como genial. Decidieron establecer una única norma que los asistentes tenían que respetar a rajatabla (Dytham, 2003, pág. 83).

Gamestorming juegos de base

Los juegos de base son actividades sencillas y de breve duración que pueden realizarse a partir de recursos como post-its y tarjetas de cartón y sirven para planificar tareas, introducir nuevas temáticas, tomar decisiones rápidas $y$, en general, dinamizar las actividades cotidianas (Scolari, 2014, pág. 83).

\section{Gamestorming juegos de apertura}

Los juegos de apertura están centrados en generar ideas, proponer soluciones a problemas, generar conexiones entre personas, optimizar el tiempo. Resultan ser útiles, sobre todo, en aquellos casos en que un equipo de trabajo queda estancado delante de la solución de un problema.

Estos juegos se realizan para desarrollar la creatividad, ingeniosidad y buscar soluciones a problemas y generar opiniones diversas (Scolari, 2012, pág. 83).

Gamestorming juegos para explorar

Los juegos que exploran permiten deshacer patrones y preconceptos a través de la combinación e interpretación de ideas nuevas. Suelen ser utilizados para optimizar informaciones, contextualizar problemas, profundizar temáticas complejas, individuar puntos débiles y oportunidades, repensar y remodelar estrategias (Scolari, 2012, pág. 83). 


\section{Partnering}

Al contrario de la clase magistral, en el partnering, el profesor da instrumentos para que los alumnos puedan contestar a las preguntas. El docente, en su rol de diseñador del aprendizaje y controlador del proceso de calidad, tiene el mismo peso que el alumno y las clases se convierten en una ocasión de intercambio (Prensky, 2012, pág. 83).

\section{Debriefing}

El debriefing es una técnica que se inscribe dentro de la filosofía de partnering y consiste en una reflexión a posteriori sobre las tareas realizadas por los alumnos. El debriefing promociona el aprendizaje a partir de las acciones y del grupo y, a la vez, permite reflexionar sobre la misma experiencia de aprendizaje (Prensky, 2012, pág. 83).

\section{Alternate reality games ( $A R G$ )}

Los juegos de realidad alternativa son actividades que se pueden realizar en el mundo virtual y real y se diferencian de los juegos de rol porque los participantes no han de construir una identidad alternativa, sino que han de ser ellos mismos. Estas actividades son ideales para trabajar aspectos narrativos y de territorio, fomentando la cultura del diseño y el sentimiento de comunidad (Prensky, 2012, pág. 83).

La educación en general se ha convertido en el centro de las políticas públicas luego de muchos años de abandono a la educación. Mediante la aprobación de la ley orgánica de educación Superior LOES) en octubre del 2010 se consideró la importancia de crear universidades en una nueva sociedad del conocimiento cambiante y estas transformaciones son las que se necesita para un desarrollo social y económico del país. Los resultados han sido favorables en cuanto a la formación de docentes, infraestructura y sistema de evaluación, queda mucho en el tintero para expresar que la calidad de la educación y el compromiso de los estudiantes es necesario educar a los ciudadanos en disciplinarse en mejorar los ambientes de aprendizaje para llegar a una práctica en el aula con nuevas pedagogías innovadas.

Fomentar el compromiso de auto superación, metas que requieren cumplir, estrategias de proyectos de vida. El docente en la actualidad debe ser un consejero y guía de los estudiantes, respetar los criterios de otros, la autoestima, hermandad, igualdad, respeto a todos los ciudadanos sin exclusión de ninguna índole.

\section{Conclusiones}

La Universidad actual es efectiva, productiva, generadora de humanidad y de compromisos sociales, es el resultado de formar educadores y profesionales que estén dispuestos a construir un futuro mejor, que los sueños sean convertidos en realidad y de vencer muchas dificultades hasta alcanzar los objetivos propuestos ñ por ello un estudiante de la universidad tiene como referentes sociales a los padres que son los guiadores del futuro.

Los propios estudiantes son los arquitectos de su vida, la escuela y la universidad construye espacio para impartir saberes, pero el camino que debe tomar la decisión absoluta es de los estudiantes. Se necesita autoridad pedagógica, educar en valores y ética, el respeto como principio de gran valor para la interacción con cada uno de los grupos y en las diversas edades. 


\section{Referencias bibliográficas}

Bramwell, M. A. (2015). Cambios en la política educativa en Ecuador desde el 2000. Education for all Global Monitoring, Report 2015, 2.

Dytham, A. K. (12 de enero de 2003). Klein-Dytham Architecture. Obtenido de www.pecha-kucha.org: http://www.pechakucha.org/

López, E. I. (2011). Las actuales propuestas y desafíos en Educación: El caso ecuatoriano. Educ. Soc., Campinas, v. 32, n. 115, p. 373-391, abr.-jun. 2011.

Noro, P. J. (12 de octubre de 2015). La escuela tenía entidad y representación social. La escuela del pasado a la escuela del futuro. Buenos Aires, Argentina: OIUDSMA.

Prensky, M. (2012). \#UPF2020 Diseñar la Universidad del Futuro. Teaching Digital Natives. Partnering for Real Learning. Thousand Oaks (CA): Corwin Press, 83.

Ramírez, R. (2013). Tercera ola de transformación de la Educación Superior en Ecuador. SENESCYT, Quito.

Scolari, C. A. (2014). \#UPF2020 Diseñar la Universidad del futuro. Quórum, UPF Barcelona School of Management. Espacio monográfico. Miradas transversales, voces y ciencia. Obtenido de https://quorum.bsm.upf.edu/temas/upf2020disenar-la-universidad-del-futuro/

The Flipped Classroom. (17 de septiembre de 2015). La innovación educativa. Obtenido de www.theflippedclassroom.es: http://www.theflippedclassroom.es/what-is- innovacion-educativa/

Unesco. (15 de agosto de 2015). http://wwwefareport.unesco.org. Recuperado el 20 de junio de 2016, de http://wwwefareport.unesco.org: http://es.unesco.org/gemreport/sites/gem-report/files/UNGA_PR_sp.pdf

UNESCOPRESS. (08 de 09 de 2014). http://www.unesco.org. Recuperado el 18 de 06 de 2016, de http://www.unesco.org: http://www.unesco.org/new/es/mediaservices/single-

view/news/unesco_sustainable_development_begins_with_education/ \#.V2yTs tLhCM8 